\title{
ALMOST SPLIT SEQUENCES FOR COMPLEXES VIA RELATIVE HOMOLOGY
}

\author{
GANG YANG
}

\begin{abstract}
We use methods of relative homological algebra on the category $\mathcal{C}(\bmod \Lambda)$, of complexes of finitely generated modules over an artin algebra $\Lambda$, to give some characterizations of almost split sequences.
\end{abstract}

\section{Introduction}

Throughout this article, we let $\Lambda$ be an $R$-algebra which is a finitely generated $R$-module, where $R$ is a commutative artin ring. We denote by $\bmod \Lambda$ the category of finitely generated left $\Lambda$-modules, and $J(\Lambda)$ the Jacboson radical of $\Lambda$.

The concept of an almost split sequence (or Auslander-Reiten sequence) in $\bmod \Lambda$ was introduced by Auslander and Reiten [3], and it plays an important role in the study of representation theory. The theory of almost split sequences developed further, for example, some results for subcategories of $\bmod \Lambda$ $[5,14,18]$, the corresponding almost split exact triangles were introduced by Happel [11, 12], and studied extensively $[7,13,15,19]$. The existence of almost split sequences was studied in more general abelian categories [16, 17], and in monomorphism categories [21]. In [19], by letting the Nakayama functor act degree-wise, Salarian and Vahed defined a translation $\tau$ in the category $\mathcal{C}(\bmod \Lambda)$ of complexes of finitely generated left $\Lambda$-modules. They investigated the existence of almost split sequences in $\mathcal{C}^{b}(\bmod \Lambda)$, and proved that for any bounded non-projective complex $X$ with local endomorphism ring there exists an almost split sequence ending in $X$.

In his thesis, Verdier introduced the notion of a Cartan-Eilenberg (abbreviated C-E) injective complex (Definition 4.6.1 of [20]) and considered the so called C-E injective and C-E projective resolutions of complexes. In [9], using the ideas of Verdier, Enochs further showed that C-E resolutions can be defined in terms of C-E injective preenvelopes (left approximation) and C-E

Received December 9, 2014.

2010 Mathematics Subject Classification. 16G10, 16G70, 18E10, 18G25, 18G35.

Key words and phrases. C-E exact sequences, Auslander-Reiten translations, almost split sequences, additive subbifunctors, non-degenerated bilinear forms. 
projective precovers (right approximation), respectively, and then a new homological functor $\overline{\operatorname{Ext}}^{1}(-,-)$ introduced, which is in fact an additive subbifunctor of $\operatorname{Ext}^{1}(-,-)$ in the sense of $[6,8]$. Since relative homological algebra was already applied well to study the representation theory of artin algebras in the category of modules (see $[6,8,22]$ ), we are motivated to study almost split sequences in the category of complexes and give some characterizations of an almost split sequence under the additive subbifunctor $\overline{\operatorname{Ext}}^{1}(-,-)$, which we denote by $\xi \mathrm{xt}^{1}(-,-)$ in the present paper.

In Section 2, we investigate some homological properties of complexes relating to $\mathrm{C}$-E projectives and $\mathrm{C}-\mathrm{E}$ injectives in the category $\mathcal{C}(\bmod \Lambda)$. Then in Section 3 , we apply the obtained results to characterize almost split sequences in $\mathcal{C}(\bmod \Lambda)$. In particular, we find out an almost split sequence in $\mathcal{C}(\bmod \Lambda)$ ending in a given $\mathrm{C}-\mathrm{E}$ projective but non-projective complex, Proposition 3.7, and then give some characterizations of an almost split sequence ending in a given non-C-E projective complex, Theorems 3.11 and 3.12 .

Throughout the paper, all modules are finitely generated left unitary $\Lambda$ modules, unless stated specially. To every complex

$$
X=: \cdots \rightarrow X_{n+1} \stackrel{d_{n+1}}{\longrightarrow} X_{n} \stackrel{d_{n}}{\longrightarrow} X_{n-1} \rightarrow \cdots,
$$

the $n$th cycle of $X$ is defined as $\operatorname{Ker}\left(d_{n}\right)$ and is denoted by $\mathrm{Z}_{n}(X)$, the $n$th boundary is defined as $\operatorname{Im}\left(d_{n+1}\right)$ and is denoted by $\mathrm{B}_{n}(X)$. We use $\mathrm{Z}(X)$, $\mathrm{B}(X) \subseteq X$ to denote the subcomplexes of cycles and boundaries of $X$. The suspension of $X$, denoted by $\Sigma X$, is the complex given by $(\Sigma X)_{n}=X_{n-1}$ and $d_{n}^{\Sigma X}=-d_{n-1}^{X}$. A complex $X$ is bounded above (resp. bounded below) if $X_{i}=0$ for all but finitely many $i>0$ (resp. finitely many $i<0$ ). A complex $X$ is called bounded if it is both bounded above and bounded below. We denote by $\mathcal{C}(\bmod \Lambda)$ the category of complexes of finitely generated left $\Lambda$-modules and $\mathcal{C}^{b}(\bmod \Lambda)$ the full subcategory of bounded complexes.

\section{Some homological theories on $\mathcal{C}(\bmod \Lambda)$}

In this section, we will investigate some basic homological properties of complexes relating to $\mathrm{C}$-E projective and $\mathrm{C}-\mathrm{E}$ injective complexes for our use later. Recall that a sequence of complexes $0 \rightarrow U \rightarrow V \rightarrow W \rightarrow 0$ is exact if all the sequences of modules $0 \rightarrow U_{n} \rightarrow V_{n} \rightarrow W_{n} \rightarrow 0$ are exact for $n \in \mathbb{Z}$. The following lemma follows easily from the snake lemma (see [9] for the detail).

Lemma 2.1. Let $0 \rightarrow U \rightarrow V \rightarrow W \rightarrow 0$ be an exact sequence of complexes in $\mathcal{C}(\bmod \Lambda)$. Consider the following induced sequences of complexes:

(1) $0 \rightarrow \mathrm{Z}(U) \rightarrow \mathrm{Z}(V) \rightarrow \mathrm{Z}(W) \rightarrow 0$,

(2) $0 \rightarrow \mathrm{B}(U) \rightarrow \mathrm{B}(V) \rightarrow \mathrm{B}(W) \rightarrow 0$

(3) $0 \rightarrow U / \mathrm{Z}(U) \rightarrow V / \mathrm{Z}(V) \rightarrow W / \mathrm{Z}(W) \rightarrow 0$,

(4) $0 \rightarrow U / \mathrm{B}(U) \rightarrow V / \mathrm{B}(V) \rightarrow W / \mathrm{B}(W) \rightarrow 0$. 
If any one of the induced sequences (1)-(4) is exact, then all of (1)-(4) are exact.

Based upon the above lemma, we have the following definition (see [9]).

Definition 2.2. An exact sequence of complexes $\zeta: 0 \rightarrow U \rightarrow V \rightarrow W \rightarrow 0$ is called Cartan-Eilenberg (abbreviated C-E) exact if any one of the induced sequences (1)-(4) in Lemma 2.1 by $\zeta$ is exact.

If $M$ is a $\Lambda$-module, then $M$ can be regarded as a complex concentrated at $n$th degree, and we will denote this complex by $s_{n}(M)$. Similarly, we denote the complex $t_{n}(M)=: \cdots \rightarrow 0 \rightarrow M \stackrel{=}{\rightarrow} M \rightarrow 0 \rightarrow \cdots$ with $M$ in the $n$-th and $(n-1)$-th degrees. The following examples show that an exact sequence of complexes may not be $\mathrm{C}-\mathrm{E}$ exact, and at the same time, there exists lots of C-E exact sequences in $\mathcal{C}(\bmod \Lambda)$.

Example 2.3. Let $M$ be a $\Lambda$-module. Then the sequence of complexes $0 \rightarrow$ $s_{n}(M) \rightarrow t_{n+1}(M) \rightarrow s_{n+1}(M) \rightarrow 0$ is clearly exact but not C-E exact in $\mathcal{C}(\bmod \Lambda)$.

Example 2.4. Let $K \subsetneq L$ be two submodules of a $\Lambda$-module $M$. Then the following sequence of complexes is exact but not $\mathrm{C}-\mathrm{E}$ exact in $\mathcal{C}(\bmod \Lambda)$ with canonical injections and surjections.

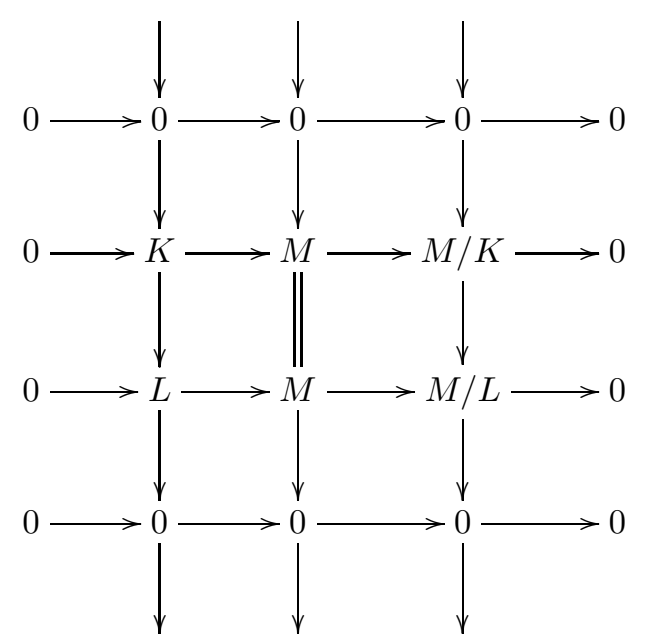

Example 2.5. If $0 \rightarrow L \rightarrow M \rightarrow N \rightarrow 0$ is an exact sequence of $\Lambda$-modules, then the sequences of complexes $0 \rightarrow s_{n}(L) \rightarrow s_{n}(M) \rightarrow s_{n}(N) \rightarrow 0$ and $0 \rightarrow t_{n}(L) \rightarrow t_{n}(M) \rightarrow t_{n}(N) \rightarrow 0$ are clearly C-E exact in $\mathcal{C}(\bmod \Lambda)$.

Example 2.6. An exact sequence of complexes $0 \rightarrow U \rightarrow V \rightarrow W \rightarrow 0$ with $W$ an acyclic complex is always $\mathrm{C}-\mathrm{E}$ exact in $\mathcal{C}(\bmod \Lambda)$ by $[9, \operatorname{Prop} .10 .1]$. 
The following definition is essentially Verdier's [20], which is explicitly stated and further considered by Enochs [9].

Definition 2.7. A complex $P \in \mathcal{C}(\bmod \Lambda)$ is called a $\mathrm{C}-\mathrm{E}$ projective complex if $P, \mathrm{Z}(P), \mathrm{B}(P)$, and $\mathrm{Z}(P) / \mathrm{B}(P)$ are all complexes of projective $\Lambda$-modules. Dually, a complex $I \in \mathcal{C}(\bmod \Lambda)$ is called a $\mathrm{C}$-E injective complex if $I, \mathrm{Z}(I)$, $\mathrm{B}(I)$, and $\mathrm{Z}(I) / \mathrm{B}(I)$ are all complexes of injective $\Lambda$-modules.

Assume that $M$ is a projective $\Lambda$-module. Then it is not difficult to show that $t_{n}(M)$ is a projective complex. In fact, a complex $P$ is a projective complex if and only if $P=\oplus_{n \in \mathbb{Z}} t_{n}\left(M_{n}\right)$ for some projective $\Lambda$-modules $M_{n}$. By [9, Prop. 3.4], a complex $P$ is a C-E projective complex if and only if $P=P^{\prime} \oplus P^{\prime \prime}$ where $P^{\prime}$ is a projective complex and where $P^{\prime \prime}$ is a complex of projective modules with all differentials being zero, that is, $P^{\prime \prime}=\oplus_{n \in \mathbb{Z}} s_{n}\left(L_{n}\right)$ for some projective $\Lambda$-modules $L_{n}$. Dually, a complex $I$ is C-E injective if and only if $I=I^{\prime} \oplus I^{\prime \prime}$, where $I^{\prime}$ is an injective complex and where $I^{\prime \prime}$ is a complex of injective modules with all differentials being zero.

Notation 2.8. Let $\xi$ be the class of all short $\mathrm{C}$-E exact sequences in $\mathcal{C}(\bmod \Lambda)$. We denote by $\mathcal{P}(\xi)$ the full subcategory of $\xi$-projective objects (see [6]), that is, all complexes $P \in \mathcal{C}(\bmod \Lambda)$ such that if $0 \rightarrow U \rightarrow V \rightarrow W \rightarrow 0 \in \xi$, the sequence

$$
0 \rightarrow \operatorname{Hom}_{\mathcal{C}}(P, U) \rightarrow \operatorname{Hom}_{\mathcal{C}}(P, V) \rightarrow \operatorname{Hom}_{\mathcal{C}}(P, W) \rightarrow 0
$$

is exact, where $\operatorname{Hom}_{\mathcal{C}}(-,-)$ is $\operatorname{Hom}_{\mathcal{C}(\bmod \Lambda)}(-,-)$ for simplicity. Dually, we have the full subcategory $\mathcal{I}(\xi)$ of $\xi$-injective objects.

Proposition 2.9. A complex $P \in \mathcal{C}(\bmod \Lambda)$ is in the subcategory $\mathcal{P}(\xi)$ if and only if it is a $C$-E projective complex. Dually, a complex $I \in \mathcal{C}(\bmod \Lambda)$ is in the subcategory $\mathcal{I}(\xi)$ if and only if it is a $C$-E injective complex.

Proof. We need only to show the first part since proof of the second part is dual. If $P$ is a C-E projective complex, then one has a decomposition

$$
P=\left(\oplus_{n \in \mathbb{Z}} t_{n}\left(K_{n}\right)\right) \bigoplus\left(\oplus_{n \in \mathbb{Z}} s_{n}\left(L_{n}\right)\right),
$$

where $K_{n}$ and $L_{n}$ are projective $\Lambda$-modules. Clearly, $\operatorname{Hom}_{\mathcal{C}}\left(\oplus_{n \in \mathbb{Z}} t_{n}\left(K_{n}\right),-\right)$ exacts any short exact sequence since each $t_{n}\left(K_{n}\right)$ is a projective complex. For any short C-E exact sequence $0 \rightarrow U \rightarrow V \rightarrow W \rightarrow 0$, we note that any morphism $s_{n}\left(L_{n}\right) \rightarrow W$ is just a morphism $s_{n}\left(L_{n}\right) \rightarrow \mathrm{Z}(W)$, such morphism can be lifted to $s_{n}\left(L_{n}\right) \rightarrow \mathrm{Z}(V)$ since the sequence $0 \rightarrow \mathrm{Z}(U) \rightarrow \mathrm{Z}(V) \rightarrow$ $\mathrm{Z}(W) \rightarrow 0$ is exact by the hypothesis and $L_{n}$ is a projective $\Lambda$-module. This implies clearly that $\operatorname{Hom}_{\mathcal{C}}\left(s_{n}\left(L_{n}\right),-\right)$ exacts the C-E exact sequence $0 \rightarrow U \rightarrow$ $V \rightarrow W \rightarrow 0$, and so does $\operatorname{Hom}\left(\oplus_{n \in \mathbb{Z}} s_{n}\left(L_{n}\right),-\right)$. Thus we show that $P=$ $\left(\oplus_{n \in \mathbb{Z}} t_{n}\left(K_{n}\right)\right) \bigoplus\left(\oplus_{n \in \mathbb{Z}} s_{n}\left(L_{n}\right)\right)$ belongs to $\mathcal{P}(\xi)$.

Conversely, let $P \in \mathcal{P}(\xi)$. We first take an epimorphism $Q \rightarrow P / \mathrm{Z}(P)$ with $Q$ a projective complex in $\mathcal{C}(\bmod \Lambda)$, and lift it to a morphism $q: Q \rightarrow$ $P$. Then we have a morphism $\mathrm{Z}(Q) \rightarrow \mathrm{Z}(P)$ which is induced by $q$. Set 
$C=\operatorname{Coker}(\mathrm{Z}(Q) \rightarrow \mathrm{Z}(P))$ and let $Q^{\prime} \rightarrow C$ be an epimorphism such that $Q^{\prime}=\oplus_{n \in \mathbb{Z}} s_{n}\left(Q_{n}^{\prime}\right)$ for some projective $\Lambda$-modules. Again we lift $Q^{\prime} \rightarrow C$ to a morphism $q^{\prime}: Q^{\prime} \rightarrow \mathrm{Z}(P)$. Now, we have an obvious morphism $\alpha: Q \oplus Q^{\prime} \rightarrow P$ which is given by $q$ and $q^{\prime}$. Obviously, $Q \oplus Q^{\prime}$ is a C-E projective complex, and it can be checked easily that the induced morphisms $\mathrm{Z}(\alpha): \mathrm{Z}\left(Q \oplus Q^{\prime}\right) \rightarrow \mathrm{Z}(P)$ and $\left(Q \oplus Q^{\prime}\right) / \mathrm{Z}\left(Q \oplus Q^{\prime}\right) \rightarrow P / \mathrm{Z}(P)$ are both epimorphisms. This implies that $\alpha: Q \oplus Q^{\prime} \rightarrow P$ is a C-E projective precover of $P$ ([9, Prop. 3.6]). Therefore, it follows from [9, Prop. 5.4] that there is a C-E exact sequence

$$
0 \rightarrow K \rightarrow Q \oplus Q^{\prime} \rightarrow P \rightarrow 0 .
$$

We get that $P$ is a direct summand of $Q \oplus Q^{\prime}$ since the sequence

$$
0 \rightarrow \operatorname{Hom}_{\mathcal{C}}(P, K) \rightarrow \operatorname{Hom}_{\mathcal{C}}\left(P, Q \oplus Q^{\prime}\right) \rightarrow \operatorname{Hom}_{\mathcal{C}}(P, P) \rightarrow 0
$$

is exact, and so $P$ is a $\mathrm{C}-\mathrm{E}$ projective complex. This completes the proof.

Let $\mathcal{X}$ be a given full subcategory of the category $\mathcal{C}(\bmod \Lambda)$. For each pair $U$ and $W$ in $\mathcal{C}(\bmod \Lambda)$, we define a class $F_{\mathcal{X}}(W, U)\left(\operatorname{resp} . F^{\mathcal{X}}(W, U)\right)$ of all short exact sequence

$$
0 \rightarrow U \rightarrow V \rightarrow W \rightarrow 0
$$

of complexes such that the sequence

$$
\begin{gathered}
0 \rightarrow \operatorname{Hom}_{\mathcal{C}}(X, U) \rightarrow \operatorname{Hom}_{\mathcal{C}}(X, V) \rightarrow \operatorname{Hom}_{\mathcal{C}}(X, W) \rightarrow 0 \\
\left(\text { resp. } 0 \rightarrow \operatorname{Hom}_{\mathcal{C}}(W, X) \rightarrow \operatorname{Hom}_{\mathcal{C}}(V, X) \rightarrow \operatorname{Hom}_{\mathcal{C}}(U, X) \rightarrow 0\right)
\end{gathered}
$$

remains exact for any complex $X \in \mathcal{X}$. Then $F_{\mathcal{X}}(-,-)$ and $F^{\mathcal{X}}(-,-)$ become additive subbifunctors of the usual functor $\operatorname{Ext}_{\mathcal{C}}^{1}(-,-)$ (see [6, Prop. 1.7]). We have the following connection between the two different methods of constructing subbifunctors of $\operatorname{Ext}_{\mathcal{C}}^{1}(-,-)$, compare to [6, Prop. 1.8].

Proposition 2.10. $F_{\mathcal{P}(\xi)}(-,-)=\xi=F^{\mathcal{I}(\xi)}(-,-)$.

Proof. We need only to show the first equality since proof of the second one is dual. It follows easily from Proposition 2.9 that $\xi \subseteq F_{\mathcal{P}(\xi)}(-,-)$. Now let $0 \rightarrow U \rightarrow V \rightarrow W \rightarrow 0$ be a short exact sequence in $F_{\mathcal{P}(\xi)}(W, U)$. Then the sequence

$$
0 \rightarrow \operatorname{Hom}_{\mathcal{C}}(P, U) \rightarrow \operatorname{Hom}_{\mathcal{C}}(P, V) \rightarrow \operatorname{Hom}_{\mathcal{C}}(P, W) \rightarrow 0
$$

is exact for each $P \in \mathcal{P}(\xi)$. In particular, the sequence

$$
0 \rightarrow \operatorname{Hom}_{\mathcal{C}}\left(s_{n}(\Lambda), U\right) \rightarrow \operatorname{Hom}_{\mathcal{C}}\left(s_{n}(\Lambda), V\right) \rightarrow \operatorname{Hom}_{\mathcal{C}}\left(s_{n}(\Lambda), W\right) \rightarrow 0
$$

is exact since $s_{n}(\Lambda)$ is a C-E projective complex for each $n \in \mathbb{Z}$. Thus we have an exact sequence

$$
0 \rightarrow \mathrm{Z}_{n}(U) \rightarrow \mathrm{Z}_{n}(V) \rightarrow \mathrm{Z}_{n}(W) \rightarrow 0
$$

since $\operatorname{Hom}_{\mathcal{C}}\left(s_{n}(\Lambda), X\right) \cong \mathrm{Z}_{n}(X)$ for any complex $X$. This implies that the sequence $0 \rightarrow U \rightarrow V \rightarrow W \rightarrow 0$ is $\mathrm{C}$-E exact, and so $F_{\mathcal{P}(\xi)}(-,-) \subseteq \xi$, and we have $F_{\mathcal{P}(\xi)}(-,-)=\xi$, as desired. 
Remark 2.11. An exact sequence $0 \rightarrow U \rightarrow V \rightarrow W \rightarrow 0$ in the category $\mathcal{C}(\bmod \Lambda)$ is $\mathrm{C}-\mathrm{E}$ exact if and only if

$$
0 \rightarrow \operatorname{Hom}_{\mathcal{C}}\left(s_{n}(\Lambda), U\right) \rightarrow \operatorname{Hom}_{\mathcal{C}}\left(s_{n}(\Lambda), V\right) \rightarrow \operatorname{Hom}_{\mathcal{C}}\left(s_{n}(\Lambda), W\right) \rightarrow 0
$$

is exact for each $n \in \mathbb{Z}$, if and only if

$$
0 \rightarrow \operatorname{Hom}_{\mathcal{C}}\left(W, s_{n}(I)\right) \rightarrow \operatorname{Hom}_{\mathcal{C}}\left(V, s_{n}(I)\right) \rightarrow \operatorname{Hom}_{\mathcal{C}}\left(U, s_{n}(I)\right) \rightarrow 0
$$

is exact for each $n \in \mathbb{Z}$ because of $\operatorname{Hom}_{\mathcal{C}}\left(X, s_{n}(I)\right) \cong \operatorname{Hom}_{\Lambda}\left(X_{n} / \mathrm{B}_{n}(X), I\right)$ for any complex $X$, where $I$ is an injective cogenerator for the category $\bmod \Lambda$.

A subcategory $\mathcal{X}$ is said to be projectively resolving, if it contains all projective objects and for any short exact $0 \rightarrow X^{\prime} \rightarrow X \rightarrow X^{\prime \prime} \rightarrow 0$ with $X^{\prime \prime} \in \mathcal{X}$, $X^{\prime} \in \mathcal{X}$ if and only if $X \in \mathcal{X}$. The following example implies that the subcategory $\mathcal{P}(\xi)$ of all C-E projective complexes is not projectively resolving.

Example 2.12. Let $\Lambda=k[x] /\left(x^{2}\right)$ be an artin algebra, where $k$ is a field, $x$ an indeterminant, and $\left(x^{2}\right)$ is the ideal generated by $x^{2}$.

(1) Consider the following commutative diagram

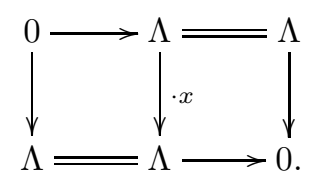

It is obvious that the middle term $X$, of the short exact sequence $0 \rightarrow s_{n}(\Lambda) \rightarrow X \rightarrow s_{n+1}(\Lambda) \rightarrow 0$, is not C-E projective though $s_{n}(\Lambda)$ and $s_{n+1}(\Lambda)$ are C-E projective, where $X=: \cdots \rightarrow 0 \rightarrow \Lambda \stackrel{\cdot x}{\rightarrow} \Lambda \rightarrow$ $0 \rightarrow \cdots$. Thus the subcategory $\mathcal{P}(\xi)$ of $\mathrm{C}-\mathrm{E}$ projective complexes is not closed under extensions.

(2) Consider the following commutative diagram

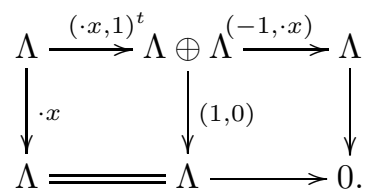

Then one can construct an exact sequence

$$
0 \rightarrow X \rightarrow t_{n+1}(\Lambda) \oplus s_{n+1}(\Lambda) \rightarrow s_{n+1}(\Lambda) \rightarrow 0
$$

with the first term $X$ as in (1) being non-C-E projective and the last two terms $t_{n+1}(\Lambda) \oplus s_{n+1}(\Lambda)$ and $s_{n+1}(\Lambda)$ being C-E projective. Thus the subcategory $\mathcal{P}(\xi)$ of C-E projective complexes is not closed under kernels of epimorphisms.

Clearly, the subcategory $\mathcal{P}(\xi)$ contains all projective complexes, but it is not projectively resolving as we have shown above. The following proposition implies that the subcategory $\mathcal{P}(\xi)$ admits a property similar to that of a projectively resolving class. 
Proposition 2.13. Let $0 \rightarrow U \rightarrow V \rightarrow W \rightarrow 0$ be a $C$-E exact sequence in $\mathcal{C}(\bmod \Lambda)$. If $W$ is $C$-E projective, then $U$ is $C$ - $E$ projective if and only if $V$ is $C$ - $E$ projective. Dually, if $U$ is $C$ - $E$ injective, then $W$ is $C$ - $E$ injective if and only if $V$ is $C$-E injective.

Proof. By the hypothesis, one gets that the induced sequences

$$
0 \rightarrow \mathrm{Z}(U) \rightarrow \mathrm{Z}(V) \rightarrow \mathrm{Z}(W) \rightarrow 0
$$

and

$$
0 \rightarrow \mathrm{B}(U) \rightarrow \mathrm{B}(V) \rightarrow \mathrm{B}(W) \rightarrow 0
$$

are exact, and so the sequence

$$
0 \rightarrow \mathrm{Z}(U) / \mathrm{B}(U) \rightarrow \mathrm{Z}(V) / \mathrm{B}(V) \rightarrow \mathrm{Z}(W) / \mathrm{B}(W) \rightarrow 0
$$

is exact, then the result follows easily.

Using [19, Lemma 2.1], one gets that any endomorphism of $W \in \mathcal{C}(\bmod \Lambda)$ factoring through some projective complex is nilpotent if $W$ is an indecomposable non projective complex, and we extends this result to the case relating to C-E projective complexes.

Proposition 2.14. Assume that $W \in \mathcal{C}(\bmod \Lambda)$ is an indecomposable non- $C-E$ projective complex. Then any endomorphism $f \in \operatorname{End}_{\mathcal{C}}(W)$ factoring through some $C$-E projective complex is nilpotent.

Proof. Let $f \in \operatorname{End}_{\mathcal{C}}(X)$ factor through some C-E projective complex. Suppose that $k$-th power $f^{k} \neq 0$ for any integer $k \geq 1$. By [9, Prop. 3.6] and [19, Prop. 3.6], we get easily that $W$ has a C-E projective precover $g: Q \rightarrow W$ in $\mathcal{C}(\bmod \Lambda)$, and then we have a $\mathrm{C}$-E exact sequence $0 \rightarrow K \rightarrow Q \rightarrow W \rightarrow 0$ with $K=\operatorname{Ker}(g)$ [9, Prop. 5.4]. Since $f$ factors through some C-E projective complex, there is a morphism $h: W \rightarrow Q$ such that $f=g h$, that is, we have the following commutative diagram with $f_{n}=g_{n} h_{n}$ for each $n \in \mathbb{Z}$.

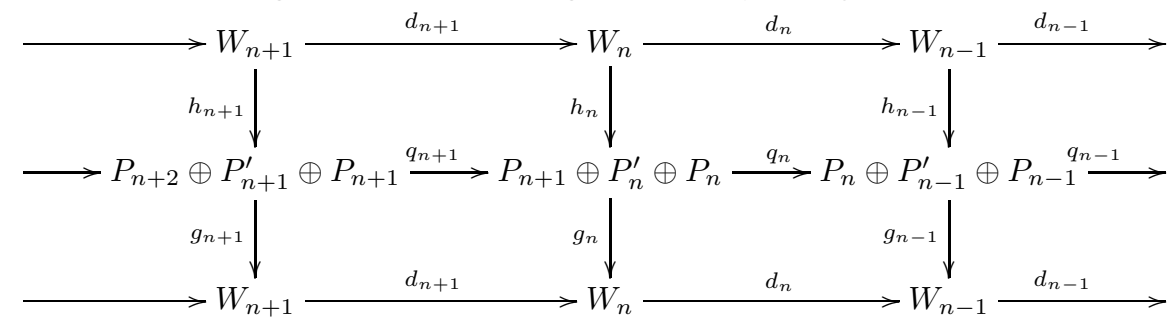

where $Q_{n}=P_{n+1} \oplus P_{n}^{\prime} \oplus P_{n}, q_{n}=\left(\begin{array}{ccc}0 & 0 & i d_{P_{n}} \\ 0 & 0 & 0 \\ 0 & 0 & 0\end{array}\right), h_{n}=\left(\begin{array}{c}p_{n} \\ p_{n}^{\prime} \\ p_{n-1} d_{n}\end{array}\right)$ for each $n \in \mathbb{Z}$. Since $\Lambda$ is an artin algebra, there exists an integer $l>1$ such that $l$-th power $J^{l}(\Lambda)=0$. Thus we deduce that $\operatorname{Im}(h) \nsubseteq J(\Lambda) Q$ since $f^{k} \neq 0$ for any integer $k \geq 1$. In particular, there is an integer $n \in \mathbb{Z}$ such that

$$
\operatorname{Im}\left(h_{n}\right)=\operatorname{Im}\left(\begin{array}{c}
p_{n} \\
p_{n}^{\prime} \\
p_{n-1} d_{n}
\end{array}\right) \nsubseteq J(\Lambda) P_{n+1} \oplus J(\Lambda) P_{n}^{\prime} \oplus J(\Lambda) P_{n}
$$


and $\left(f_{n}\right)^{k} \neq 0$ for some $k>l^{2}$. That is, at least one of three containments below does not hold.

(i) $\operatorname{Im}\left(p_{n}^{\prime}\right) \subseteq J(\Lambda) P_{n}^{\prime}$.

(ii) $\operatorname{Im}\left(p_{n-1} d_{n}\right) \subseteq J(\Lambda) P_{n}$.

(iii) $\operatorname{Im}\left(p_{n}\right) \subseteq J(\Lambda) P_{n+1}$.

Suppose that $\operatorname{Im}\left(p_{n}^{\prime}\right) \nsubseteq J(\Lambda) P_{n}^{\prime}$. Then it follows from [2, Prop. 2.4] that $\operatorname{Im}\left(p_{n}^{\prime}\right)$ contains a non-zero projective $\Lambda$-module $L$ which is a direct summand of $P_{n}^{\prime}$, and so of $\operatorname{Im}\left(p_{n}^{\prime}\right)$. Since $p_{n}^{\prime} d_{n+1}=0$, it is easily seen that the complex $s_{n}(L)$ is a direct summand of $W$. This contradicts to the hypothesis that $W$ is indecomposable, and so we have $\operatorname{Im}\left(p_{n}^{\prime}\right) \subseteq J(\Lambda) P_{n}^{\prime}$.

Suppose that $\operatorname{Im}\left(p_{n-1} d_{n}\right) \nsubseteq J(\Lambda) P_{n}$. Again by [2, Prop. 2.4], we get that $\operatorname{Im}\left(p_{n-1} d_{n}\right)$ contains a non-zero projective $\Lambda$-module $L$ which is a direct summand of $P_{n}$, and so of $\operatorname{Im}\left(p_{n-1} d_{n}\right)$. Since $L \subseteq \operatorname{Im}\left(p_{n-1} d_{n}\right) \subseteq \operatorname{Im}\left(p_{n-1}\right) \subseteq P_{n}$, $L$ is a direct summand of $\operatorname{Im}\left(p_{n-1}\right)$. Therefore, the complex $t_{n}(L)$ is a direct summand of $W$. This is again a contradiction. Thus we have $\operatorname{Im}\left(p_{n-1} d_{n}\right) \subseteq$ $J(\Lambda) P_{n}$.

Suppose that $\operatorname{Im}\left(p_{n}\right) \nsubseteq J(\Lambda) P_{n+1}$. If $\operatorname{Im}\left(p_{n} d_{n+1}\right) \nsubseteq J(\Lambda) P_{n+1}$, then a similar argument as the case (ii) shows that $W$ has a non-zero projective direct summand. Hence, we suppose that $\operatorname{Im}\left(p_{n} d_{n+1}\right) \subseteq J(\Lambda) P_{n+1}$. Then we deduce that

$$
\operatorname{Im}\left(\left(p_{n} d_{n+1} g_{n+1}\right)^{l-1} p_{n} d_{n+1}\right) \subseteq J^{l}(\Lambda) P_{n+1}=0,
$$

and $\operatorname{Im}\left(\left(p_{n} g_{n}\right)^{l-1} p_{n}\right) \nsubseteq J(\Lambda) P_{n+1}$, since otherwise, $\left(g_{n} p_{n}\right)^{l^{2}} \subseteq J^{l}(\Lambda) W_{n}=$ 0 , and on the other hand, it follows from the facts $\operatorname{Im}\left(p_{n}^{\prime}\right) \subseteq J(\Lambda) P_{n}^{\prime}$ and $\operatorname{Im}\left(p_{n-1} d_{n}\right) \subseteq J(\Lambda) P_{n}$ we have shown above, respectively, that $\left(g_{n} p_{n}^{\prime}\right)^{2 l}=0$ and $\left(g_{n} p_{n-1} \bar{d}_{n}\right)^{2 l}=0$. Thus we have

$$
\left(f_{n}\right)^{l^{2}}=\left(g_{n} h_{n}\right)^{l^{2}}=\left(\begin{array}{c}
\left(g_{n} p_{n}\right)^{l^{2}} \\
\left(g_{n} p_{n}^{\prime}\right)^{l^{2}} \\
\left(g_{n} p_{n-1} d_{n}\right)^{l^{2}}
\end{array}\right)=0,
$$

which is a contradiction. Again by [2, Prop. 2.4], we get that $\operatorname{Im}\left(\left(p_{n} g_{n}\right)^{l-1} p_{n}\right)$ contains a non-zero projective $\Lambda$-module $L$ which is a direct summand of $P_{n+1}$, and so of $\operatorname{Im}\left(\left(p_{n} g_{n}\right)^{l-1} p_{n}\right)$. Since $\operatorname{Im}\left(\left(p_{n} d_{n+1} g_{n+1}\right)^{l-1} p_{n} d_{n+1}\right)=0$, it is easily seen that $s_{n}(L)$ is a is a direct summand of $Q$, and then of $W$. This is a contradiction, and so we have $\operatorname{Im}\left(p_{n}\right) \subseteq J(\Lambda) P_{n+1}$.

Now we have shown that all of the cases (i), (ii) and (iii) hold, and this a contradiction. Therefore, there must exist an integer $k \geq 1$ such that $f^{k}=0$, and so $f$ is nilpotent.

\section{Almost split sequences for complexes}

In this section, we give some characterizations of an almost split sequence in the category $\mathcal{C}(\bmod \Lambda)$ of complexes. Let us first recall the relevant definitions from Auslander-Reiten theory. A morphism $f: U \rightarrow V$ is called a section if 
there exists a morphism $f^{\prime}: V \rightarrow U$ such that $f^{\prime} f=i d_{U}$. Dually, $g: V \rightarrow W$ is a retraction if there exists a morphism $g^{\prime}: W \rightarrow V$ such that $g g^{\prime}=i d_{W}$. A morphism $\alpha: U \rightarrow V$ is left almost split if $\alpha$ is not a section and if every morphism $U \rightarrow X$ that is not a section factors through $\alpha$. Dually, a morphism $\beta: V \rightarrow W$ is called right almost split if $\beta$ is not a retraction and if every morphism $Y \rightarrow W$ that is not a retraction factors through $\beta$.

Definition 3.1. An almost split sequence of complexes is an exact sequence

$$
0 \rightarrow U \stackrel{f}{\rightarrow} V \stackrel{g}{\rightarrow} W \rightarrow 0
$$

in $\mathcal{C}(\bmod \Lambda)$, where $f$ is left almost split and $g$ is right almost split.

By the same method used in the module version one can show that if $0 \rightarrow$ $U \stackrel{f}{\rightarrow} V \stackrel{g}{\rightarrow} W \rightarrow 0$ is an exact sequence in $\mathcal{C}(\bmod \Lambda)$ with $\operatorname{End}_{\mathcal{C}}(U)$ and $\operatorname{End}_{\mathcal{C}}(W)$ being local rings, then $f$ is left almost split if and only if $g$ is right almost split. Also, two almost split sequences with the same initial (or end) terms are isomorphic, see $[1,4]$.

Our first aim in this section is to characterize almost split sequences of complexes with special starting or ending terms. The following characterization of the $\mathrm{C}-\mathrm{E}$ projective and $\mathrm{C}-\mathrm{E}$ injective complexes in terms of almost split sequences is useful, which is motivated by a module version, see [6, Prop. 1.9].

Proposition 3.2. Assume that the sequence $\zeta: 0 \rightarrow U \stackrel{f}{\rightarrow} V \stackrel{g}{\rightarrow} W \rightarrow 0$ of complexes is almost split in $\mathcal{C}(\bmod \Lambda)$. Then the following assertions are equivalent.

(1) $W$ is a $C$-E projective complex.

(2) $\zeta$ is not a $C$-E exact sequence.

(3) $U$ is a $C$-E injective complex.

Proof. We need only to show $(1) \Leftrightarrow(2)$, and $(2) \Leftrightarrow(3)$ follows dually.

$(1) \Rightarrow(2)$ Let $W$ be a C-E projective complex. Since $g$ is right almost split, it follows easily that $W$ is indecomposable, and so $W$ has the form $s_{n}(P)$ or $t_{n}(P)$ for some indecomposable projective $\Lambda$-module $P$, but if $W=t_{n}(P)$, then $W$ is a projective complex, and so $\zeta$ is split, which is a contradiction. Hence, we have $W=s_{n}(P)$ with $P$ an indecomposable projective $\Lambda$-module. Now suppose that the sequence $\zeta$ is $\mathrm{C}$-E exact. Then by Proposition 2.9 , there is an exact sequence

$$
0 \rightarrow \operatorname{Hom}_{\mathcal{C}}\left(s_{n}(\Lambda), U\right) \rightarrow \operatorname{Hom}_{\mathcal{C}}\left(s_{n}(\Lambda), V\right) \rightarrow \operatorname{Hom}_{\mathcal{C}}\left(s_{n}(\Lambda), W\right) \rightarrow 0
$$

because $s_{n}(\Lambda)$ is $\mathrm{C}$-E projective, that is, the sequence

$$
0 \rightarrow \mathrm{Z}_{n}(U) \rightarrow \mathrm{Z}_{n}(V) \rightarrow \mathrm{Z}_{n}(W)=P \rightarrow 0
$$

is exact since $\operatorname{Hom}_{\mathcal{C}}\left(s_{n}(\Lambda), X\right) \cong \mathrm{Z}_{n}(X)$ for any complex $X$. But $P$ is a projective $\Lambda$-module, this implies that $P$ is a direct summand of $\mathrm{Z}_{n}(V)$. If we let $g_{n}^{\prime}: P \rightarrow \mathrm{Z}_{n}(V)$ be the canonical injection, then it is easily seen that $g^{\prime}=\left(g_{i}^{\prime}\right)_{i \in \mathbb{Z}}: W \rightarrow V$ gives a morphism of complexes satisfying $g g^{\prime}=i d_{W}$, 
where $g_{i}^{\prime}=0$ except for $i=n$. This contradicts to our condition that $\zeta$ is an almost split sequence. Thus the sequence $\zeta$ is not $\mathrm{C}$-E exact.

$(2) \Rightarrow(1)$ Assume that $\zeta$ is not a $\mathrm{C}$-E exact sequence. We claim that there must exist an epimorphism $h: s_{n}(\Lambda) \rightarrow W$ for some integer $n \in \mathbb{Z}$ such that $h$ is a retraction. If this is not the case, then any morphism $s_{n}(\Lambda) \rightarrow W$ for each $n \in \mathbb{Z}$ factors through $g$ by the hypothesis. Thus we have an exact sequence

$$
0 \rightarrow \operatorname{Hom}_{\mathcal{C}}\left(s_{n}(\Lambda), U\right) \rightarrow \operatorname{Hom}_{\mathcal{C}}\left(s_{n}(\Lambda), V\right) \rightarrow \operatorname{Hom}_{\mathcal{C}}\left(s_{n}(\Lambda), W\right) \rightarrow 0,
$$

and so the sequence

$$
0 \rightarrow \mathrm{Z}_{n}(U) \rightarrow \mathrm{Z}_{n}(V) \rightarrow \mathrm{Z}_{n}(\Lambda) \rightarrow 0
$$

is exact for each $n \in \mathbb{Z}$, which implies that $\zeta$ is $\mathrm{C}$-E exact, it contradicts to our assumption. Therefore, there exists an epimorphism $h: s_{n}(\Lambda) \rightarrow W$ for some integer $n \in \mathbb{Z}$ such that $h$ is a retraction. This implies that $W$ is a direct summand of $s_{n}(\Lambda)$, and so it is a C-E projective complex. This completes the proof.

Notation 3.3. For a complex $X \in \mathcal{C}(\bmod \Lambda)$, we let $\operatorname{Hom}_{\Lambda}(X, \Lambda)$ denote the complex in $\mathcal{C}\left(\bmod \Lambda^{\mathrm{op}}\right)$ whose $n$-th degree is $\operatorname{Hom}_{\Lambda}\left(X_{-n}, \Lambda\right)$ and the $n$-th differential is $\operatorname{Hom}_{\Lambda}\left(d_{-n+1}, \Lambda\right)$. Then $\operatorname{Hom}_{\Lambda}(-, \Lambda): \mathcal{C}(\bmod \Lambda) \rightarrow \mathcal{C}\left(\bmod \Lambda^{\mathrm{op}}\right)$ gives us a functor. Similarly, $\operatorname{Hom}_{R}(-, E): \mathcal{C}(\bmod \Lambda) \rightarrow \mathcal{C}\left(\bmod \Lambda^{\text {op }}\right)$ is also a functor, where $E$ is the injective envelope of $R / J(R)$. We denote $\operatorname{Hom}_{\Lambda}(-, \Lambda)$ by $(-)^{*}$, and $\operatorname{Hom}_{R}(-, E)$ by $\mathbf{D}(-)$. When the two functors are restricted to the category of finitely generated $\Lambda$-modules, we denote $\operatorname{Hom}_{\Lambda}(-, \Lambda)$ by $-^{*}$, and $\operatorname{Hom}_{R}(-, E)$ by $D$ with a slight distinguish.

Auslander-Reiten translations in the category of complexes are defined similarly as that in the modules category. The great importance of the AuslanderReiten translation functor is its application for determining almost split sequences.

Definition 3.4 ([19]). For any complex $X \in \mathcal{C}(\bmod \Lambda)$, take a minimal projective presentation $Q \stackrel{q}{\rightarrow} P \stackrel{p}{\rightarrow} X \rightarrow 0$ of $X$, that is, $p: P \rightarrow X$ and $q: Q \rightarrow \operatorname{Ker}(p)$ are projective covers (which always exists over $\Lambda$ by [19, Prop. 2.1]), and denote $\operatorname{Tr} X=\Sigma^{-1} \operatorname{Coker}\left(q^{*}\right)$, where $q^{*}: P^{*} \rightarrow Q^{*}$ is the morphism in $\mathcal{C}\left(\bmod \Lambda^{\mathrm{op}}\right)$ induced by $q$. The composition functors

and

$$
\tau: \mathcal{C}(\bmod \Lambda) \stackrel{\operatorname{Tr}}{\longrightarrow} \underline{\mathcal{C}}(\bmod \Lambda) \stackrel{\mathrm{D}}{\longrightarrow} \overline{\mathcal{C}}(\bmod \Lambda)
$$

$$
\tau^{-}: \mathcal{C}(\bmod \Lambda) \stackrel{\mathrm{D}}{\longrightarrow} \mathcal{C}\left(\bmod \Lambda^{\mathrm{op}}\right) \stackrel{\operatorname{Tr}}{\longrightarrow} \underline{\mathcal{C}}(\bmod \Lambda)
$$

are called Auslander-Reiten translations, where $\underline{\mathcal{C}}(\bmod \Lambda)(\operatorname{resp} . \overline{\mathcal{C}}(\bmod \Lambda))$ is the stable category of $\mathcal{C}(\bmod \Lambda)$ modulo the projective (resp. injective) complexes.

Lemma 3.5. Let $P=\Lambda$ e be an indecomposable projective $\Lambda$-module. Then $\tau\left(s_{n}(P)\right)=s_{n-1}(D(e \Lambda))$. 
Proof. We take the minimal projective presentation $t_{n-1}(P) \rightarrow t_{n}(P) \rightarrow s_{n}(P)$ $\rightarrow 0$ of $s_{n}(P)$, as explicitly shown in the diagram below.

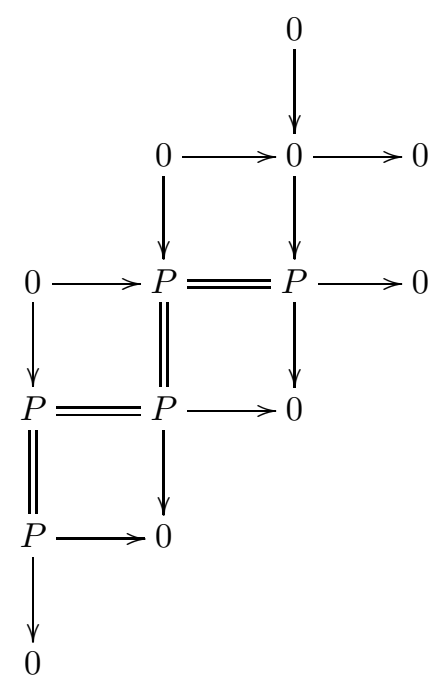

It is easily checked that $\operatorname{Coker}\left(\left[t_{n}(P)\right]^{*} \rightarrow\left[t_{n-1}(P)\right]^{*}\right)=s_{-n+2}\left(P^{*}\right)$, and so

$$
\tau\left(s_{n}(P)\right)=\mathbf{D}\left(\Sigma^{-1} s_{-n+2}\left(P^{*}\right)\right)=\mathbf{D}\left(s_{-n+1}\left(P^{*}\right)\right)=s_{n-1}(D(e \Lambda)) .
$$

Remark 3.6. The above result implies clearly that the subcategory $\mathcal{I}(\xi)$ is equal to $\tau(\mathcal{P}(\xi)) \cup \mathcal{I}(\xi)$, see [8, Prop. 2.6].

By [7, Th. 4.3], the bounded category $\mathcal{C}^{b}(\bmod \Lambda)$ admits almost split sequences, also see [19, Th. 3.1]; many almost split sequences for complexes were constructed there. In the following a particular interesting almost split sequence is given, which ends in a $\mathrm{C}$-E projective complex or equivalently starts in a $\mathrm{C}$-E injective complex. It in fact gives us a more explicit characterization of almost split sequences that are not C-E exact, compare to Proposition 3.2.

Proposition 3.7. Let $s_{n}(P)$ be an indecomposable $C$-E projective complex with $P=\Lambda e$. Then the sequence $\zeta: 0 \rightarrow s_{n-1}(D(e \Lambda)) \rightarrow V \stackrel{g}{\rightarrow} s_{n}(P) \rightarrow 0$, as explicitly shown in the following diagram,

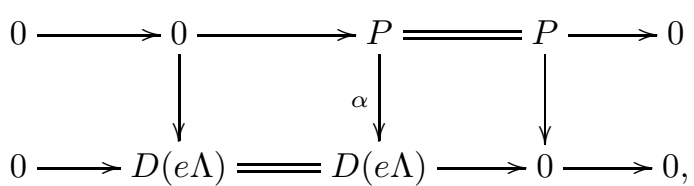

is an almost split sequence in $\mathcal{C}(\bmod \Lambda)$, where $V=(\cdots \rightarrow 0 \rightarrow \Lambda e \stackrel{\alpha}{\rightarrow} D(e \Lambda) \rightarrow$ $0 \rightarrow \cdots)$ such that $\Lambda$ e and $D(e \Lambda)$ are in the $n$-th and $(n-1)$-th degrees, respectively, and $\alpha$ is the composition of the canonical projection $\pi: \Lambda e \rightarrow$ $\Lambda e / \operatorname{rad}(\Lambda e)$ and the injective envelope $\rho: \Lambda e / \operatorname{rad}(\Lambda e) \rightarrow D(e \Lambda)$ of $\Lambda e / \operatorname{rad}(\Lambda e)$. 
Proof. Observe that $\operatorname{End}_{\mathcal{C}}\left(s_{n}(P)\right)$ and $\operatorname{End}_{\mathcal{C}}\left(s_{n-1}(D(e \Lambda))\right)$ are local rings, we need only show that $g$ is right almost split (see [1, Th. 1.13] for the module version). Let $h: X \rightarrow s_{n}(P)$ be a morphism such that it is not a retraction. We note that the induced morphism $h_{n}^{\prime}: Z_{n}(X) \rightarrow P$ by $h_{n}$ never be an epimorphism. Otherwise, the existed morphism $h_{n}^{\prime \prime}: P \rightarrow Z_{n}(X)$ satisfying $h_{n}^{\prime} h_{n}^{\prime \prime}=i d_{P}$ will provide a morphism $h^{\prime \prime}=\left(h_{i}^{\prime \prime}\right)_{i \in \mathbb{Z}}: s_{n}(P) \rightarrow V$ such that $h h^{\prime \prime}=i d_{s_{n}(P)}$, which is a contradiction, where $h_{i}^{\prime \prime}=0$ except for $i=n$. Thus we have $\operatorname{Im}\left(h_{n}^{\prime}\right) \subseteq \operatorname{rad}(P)$, and so there exists a morphism $\widetilde{h}_{n}: X_{n} / \mathrm{Z}_{n}(X) \rightarrow$ $S=\Lambda e / \operatorname{rad}(\Lambda e)$ such that the following diagram commutes.

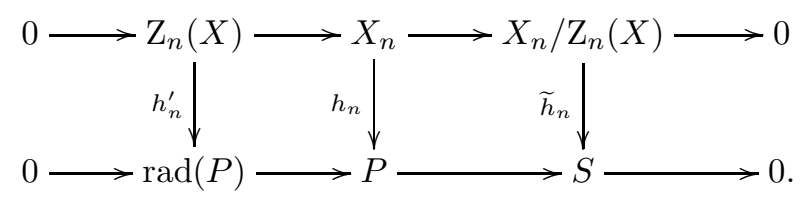

Since $D(e \Lambda)$ is an injective envelope of $S$, there is a morphism $f_{n-1}: X_{n-1} \rightarrow$ $D(e \Lambda)$ such that the following diagram is commutative.

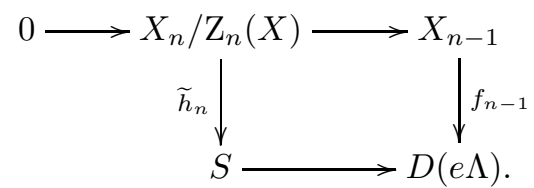

Now we have a morphism $f=\left(f_{i}\right)_{i \in \mathbb{Z}}: X \rightarrow V$ of complexes with $f_{i}=0$ except for $i=n, n-1$, and $f_{n}=h_{n}$, which satisfies $h=g f$, that is, $h$ factors through $g$. This proves that $\zeta$ is an almost split sequence.

The following result is initiated by [19, Prop. 2.2].

Lemma 3.8. Let $X \in \mathcal{C}(\bmod \Lambda)$ be a complex.

(1) If $X$ is a non-C-E projective complex with local endomorphism ring, then $\tau X$ is a non-C-E injective complex with local endomorphism ring.

(2) If $X$ is a non- $C$-E injective complex with local endomorphism ring, then $\tau^{-} X$ is a non- $C$-E projective complex with local endomorphism ring.

Proof. Since two almost split sequences with the same initial (or end) terms are isomorphic, it follows easily form Lemma 3.5 and Proposition 3.7 that $\tau$ provides a one-to-one correspondence between the classes

$$
\left\{s_{n}(\Lambda e) \mid e \text { is a primitive idempotent of } \Lambda, n \in \mathbb{Z}\right\},
$$

which contains exactly non-projective but C-E projective complexes with local endomorphism ring, and

$$
\left\{s_{n-1}(D(e \Lambda)) \mid e \text { is a primitive idempotent of } \Lambda, n \in \mathbb{Z}\right\},
$$

which contains exactly non-injective but $\mathrm{C}$-E injective complexes with local endomorphism ring. By [19, Prop. 2.2], we get that (1) holds, and (2) follows dually. 
Notation 3.9. In the next, we will denote the additive subbifunctors $F_{\mathcal{P}(\xi)}(-,-)$ and $F^{\mathcal{I}(\xi)}(-,-)$ of $\operatorname{Ext}_{\mathcal{C}}^{1}(-,-)$ by $\xi \mathrm{xt}_{\mathcal{C}}^{1}(-,-)$, which is denoted by $\overline{\operatorname{Ext}}^{1}(-,-)$ in [9].

Proposition 3.10. If $X \in \mathcal{C}^{b}(\bmod \Lambda)$ is a non-C-E projective complex with local endomorphism ring, then $\xi x t_{\mathcal{C}}^{1}(X, \tau X) \neq 0$.

Proof. One gets by Lemma 3.8 that $\tau X$ is not C-E injective. By [9, Ths. $4.1,5.6]$, it is easily seen that there exists a non-split C-E exact sequence in $\mathcal{C}^{b}(\bmod \Lambda)$

$$
0 \rightarrow \tau X \stackrel{f}{\rightarrow} V \stackrel{g}{\rightarrow} W \rightarrow 0
$$

such that $V$ is a $\mathrm{C}$-E injective complex, then $\xi \mathrm{xt}_{\mathcal{C}}^{1}(X, V)=0$, and so we get that $\xi \mathrm{xt}_{\mathcal{C}}^{1}(X, \tau X) \neq 0$ if and only if there is a morphism $X \rightarrow W$ which can not factor through $g$. But by [19, Prop. 2.4], if every morphism $X \rightarrow W$ factors through $g$, then every morphism $\tau X \rightarrow \tau X$ factors through $f$, and so the above sequence is split, which is a contradiction. Therefore, $\xi \mathrm{xt}_{\mathcal{C}}^{1}(X, \tau X) \neq 0$.

Let $\eta: 0 \rightarrow U \rightarrow V \rightarrow W \rightarrow 0$ be an extension in $\xi \operatorname{xt}_{\mathcal{C}}^{1}(W, U)$. Given two morphisms $f: U \rightarrow X$ and $g: Y \rightarrow W$, we have a push-out diagram

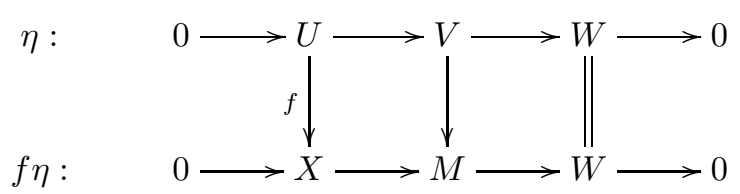

as well as a pull-back diagram

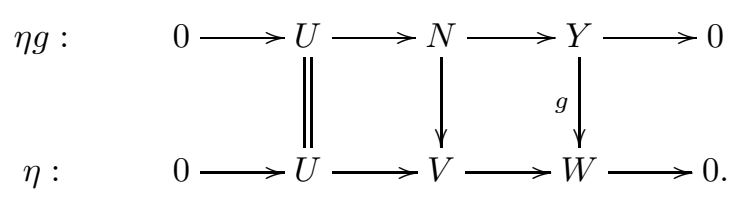

As $\xi \operatorname{xt}_{\mathcal{C}}^{1}(-,-)$ is an additive subbifunctor of $\operatorname{Ext}_{\mathcal{C}}^{1}(-,-)$, the sequences $f \eta$ and $\eta g$ are $\mathrm{C}$-E exact (see $[6,8])$, and so the abelian group $\xi \mathrm{xt}_{\mathcal{C}}^{1}(W, U)$ of equivalence classes of short $\mathrm{C}-\mathrm{E}$ exact sequences forms an $\operatorname{End}_{\mathcal{C}}(U)-\operatorname{End}_{\mathcal{C}}(W)$-bimodule under the multiplications illustrated in the above diagrams.

Given two $R$-modules $K$ and $L$ (not necessarily finitely generated). We recall from [10] that an $R$-bilinear form $\langle-,-\rangle: K \times L \rightarrow E$ is called non-degenerated provided that, for any non-zero element $k \in K$, there exists some $l \in L$ such that $\langle k, l\rangle \neq 0$, and for any non-zero element $l \in L$, there exists some $k \in K$ such that $\langle k, l\rangle \neq 0$. Observe that every $R$-linear form $\varphi: \xi \operatorname{xt}_{\mathcal{C}}^{1}(W, U) \rightarrow E$ determines, for each $X \in \mathcal{C}(\bmod \Lambda)$, two $R$-bilinear forms:

$$
\varphi\langle-,-\rangle: \xi \operatorname{xt}_{\mathcal{C}}^{1}(X, U) \times \operatorname{Hom}_{\mathcal{C} / \mathcal{P}(\xi)}(W, X) \rightarrow E ;(\eta, \underline{f}) \mapsto \varphi(\eta f),
$$

and

$$
\langle-,-\rangle_{\varphi}: \operatorname{Hom}_{\mathcal{C} / \mathcal{I}(\xi)}(X, U) \times \xi \operatorname{xt}_{\mathcal{C}}^{1}(W, X) \rightarrow E ;(\bar{f}, \eta) \mapsto \varphi(f \eta),
$$


where $E$ is an injective envelope of $R / J(R), \mathcal{C} / \mathcal{P}(\xi)$ (resp. $\mathcal{C} / \mathcal{I}(\xi)$ ) is the stable category of $\mathcal{C}(\bmod \Lambda)$ modulo the $\mathrm{C}$-E projective (resp. $\mathrm{C}-\mathrm{E}$ injective) complexes. Also, we note that if $\zeta$ is a non-zero extension in $\xi \mathrm{xt}_{\mathcal{C}}^{1}(W, U)$, then there exists always an $R$-linear form $\varphi: \xi \operatorname{xt}_{\mathcal{C}}^{1}(W, U) \rightarrow E$ such that $\varphi(\zeta) \neq 0$.

The following lemma is initiated by a result of Gabriel and Roiter [10, (9.3)], which is explicitly stated in [17, Prop. 3.1].

Lemma 3.11. Let $\zeta: 0 \rightarrow U \rightarrow V \rightarrow W \rightarrow 0$ be an almost exact sequence in $\mathcal{C}(\bmod \Lambda)$, which is $C$-E exact, and let $\varphi: \xi x t_{\mathcal{C}}^{1}(W, U) \rightarrow E$ be an $R$-linear form. If $\varphi(\zeta) \neq 0$, then the $R$-bilinear forms:

$$
\varphi\langle-,-\rangle: \xi x t_{\mathcal{C}}^{1}(X, U) \times \operatorname{Hom}_{\mathcal{C} / \mathcal{P}(\xi)}(W, X) \rightarrow E ; \quad(\eta, \underline{f}) \mapsto \varphi(\eta f),
$$

and

$$
\langle-,-\rangle_{\varphi}: \operatorname{Hom}_{\mathcal{C} / \mathcal{I}(\xi)}(X, U) \times \xi x t_{\mathcal{C}}^{1}(W, X) \rightarrow E ;(\bar{f}, \eta) \mapsto \varphi(f \eta)
$$

are both non-degenerated, for every complex $X \in \mathcal{C}(\bmod \Lambda)$.

Proof. The proof is similar to that of [17, Prop. 3.1].

Let $H: \mathcal{A} \rightarrow \operatorname{Mod} R$ and $G: \mathcal{A} \rightarrow \operatorname{Mod} R$ be covariant or contravariant $R$-linear functors, where $\mathcal{A}$ is an abelian category. Recall that a functorial monomorphism $\alpha: H \rightarrow G$ is a natural transformation with $\alpha_{X}: H(X) \rightarrow$ $G(X)$ being injective for any $X \in \mathcal{A}$. A non-zero $R$-linear form $\phi: \Lambda^{\prime} \rightarrow E$ is called almost vanishing if it vanishes on the Jacobson radical of $\Lambda^{\prime}$, where $\Lambda^{\prime}$ is an $R$-algebra, and $E$ is the injective envelope of $R / J(R)$.

In the next we will give two characterizations of an almost split sequence ending (resp. starting) in a non-C-E projective (resp. non-C-E injective) complex, which are inspired by $[17$, Ths. $3.2,3.3]$. But $\xi \mathrm{xt}_{\mathcal{C}}^{1}(-,-)$ that appears in the following characterization is a subbifunctor of $\operatorname{Ext}_{\mathcal{C}}^{1}(-,-)$.

Theorem 3.12. Let $\zeta: 0 \rightarrow \tau W \rightarrow V \rightarrow W \rightarrow 0$ be an exact sequence in $\mathcal{C}^{b}(\bmod \Lambda)$, where $W$ is a non- $C-E$ projective complex with local endomorphism ring. Then the following assertions are equivalent.

(1) $\zeta$ is an almost split sequence in $\mathcal{C}^{b}(\bmod \Lambda)$.

(2) There is a functorial monomorphism

$$
\alpha: \xi x t_{\mathcal{C}}^{1}(-, \tau W) \rightarrow D \operatorname{Hom}_{\mathcal{C} / \mathcal{P}(\xi)}(W,-)
$$

such that $\alpha_{W}(\zeta)$ is almost vanishing on $\operatorname{End}_{\mathcal{C} / \mathcal{P}(\xi)}(W)$.

(3) There is a functorial monomorphism

$$
\beta: \xi x t_{\mathcal{C}}^{1}(W,-) \rightarrow D \operatorname{Hom}_{\mathcal{C} / \mathcal{I}(\xi)}(-, \tau W)
$$

such that $\beta_{\tau W}(\zeta)$ is almost vanishing on $\operatorname{End}_{\mathcal{C} / \mathcal{I}(\xi)}(\tau W)$.

Where $\mathcal{C}$ denotes the bounded category $\mathcal{C}^{b}(\bmod \Lambda)$ of complexes, and $\mathcal{C} / \mathcal{P}(\xi)$ $($ resp. $\mathcal{C} / \mathcal{I}(\xi))$ denotes the stable category of $\mathcal{C}^{b}(\bmod \Lambda)$ modulo the $C$-E projective (resp. C-E injective) complexes. 
Proof. We will only prove $(1) \Leftrightarrow(2)$, and $(1) \Leftrightarrow(3)$ follows dually. We use an idea of proof for [17, Th. 3.2]. Note that $W$ is non-zero in $\mathcal{C} / \mathcal{P}(\xi)$ since it is not C-E projective by the hypothesis. It follows from Proposition 2.14 that every element of $\mathcal{P}(\xi)(W, W)$ is nilpotent, and so $\mathcal{P}(\xi)(W, W) \subseteq \operatorname{rad}\left(\operatorname{End}_{\mathcal{C}}(W)\right)$, where $\mathcal{P}(\xi)(W, W)$ is the two sided ideal consisting all endomorphisms which factor through C-E projective complexes. Hence, we have $\operatorname{rad}\left(\operatorname{End}_{\mathcal{C} / \mathcal{P}(\xi)}(W)\right)=$ $\operatorname{rad}\left(\operatorname{End}_{\mathcal{C}}(W)\right) / \mathcal{P}(\xi)(W, W)$ (also see [17, Lemma 2.2]).

Assume first that $\zeta$ is an almost split sequence. It is then a $\mathrm{C}$-E exact sequence by Proposition 3.2. In particular, there exists an $R$-linear form $\varphi$ : $\xi \operatorname{xt}_{\mathcal{C}}^{1}(W, \tau W) \rightarrow E$ such that $\varphi(\zeta) \neq 0$. Given a complex $X \in \mathcal{C}^{b}(\bmod \Lambda)$. By Lemma 3.11, we have a non-degenerate $R$-bilinear form

$$
\varphi\langle-,-\rangle: \xi \operatorname{xt}_{\mathcal{C}}^{1}(X, \tau W) \times \operatorname{Hom}_{\mathcal{C} / \mathcal{P}(\xi)}(W, X) \rightarrow E:(\eta, \underline{f}) \mapsto \varphi(\eta f) .
$$

This induces an $R$-linear monomorphism

$$
\alpha_{X}: \xi \operatorname{xt}_{\mathcal{C}}^{1}(X, \tau W) \rightarrow D \operatorname{Hom}_{\mathcal{C} / \mathcal{P}(\xi)}(W, X): \eta \mapsto{ }_{\varphi}\langle\eta,-\rangle .
$$

Now for any morphism $h: X \rightarrow Y$, consider the following diagram:

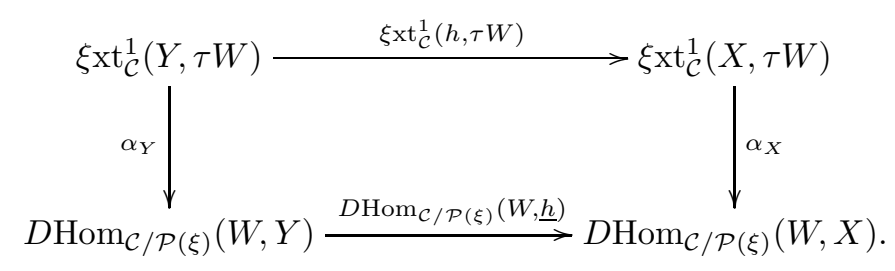

Let $\eta \in \xi \operatorname{xt}_{\mathcal{C}}^{1}(Y, \tau W)$ and $\underline{f} \in \operatorname{Hom}_{\mathcal{C} / \mathcal{P}(\xi)}(W, X)$. Then we see that

$$
\begin{aligned}
\left.\operatorname{DHom}_{\mathcal{C} / \mathcal{P}(\xi)}(W, \underline{h}) \circ \alpha_{Y}\right](\eta)(\underline{f}) & =\left[D \operatorname{Hom}_{\mathcal{C} / \mathcal{P}(\xi)}(W, \underline{h})\left(\alpha_{Y}(\eta)\right)\right](\underline{f}) \\
& =\left[\alpha_{Y}(\eta) \circ \operatorname{Hom}_{\mathcal{C} / \mathcal{P}(\xi)}(W, \underline{h})\right](\underline{f}) \\
& =\alpha_{Y}(\eta)(\underline{h} \underline{f}) \\
& =\varphi(\eta(h f)) \\
& =\varphi((\eta h) f) \\
& =\left[\alpha_{X}\left(\xi \operatorname{xt}_{\mathcal{C}}^{1}(h, \tau W)(\eta)\right)\right](\underline{f}) \\
& =\left[\alpha_{X} \circ \xi \operatorname{xt}_{\mathcal{C}}^{1}(h, \tau W)\right](\eta)(\underline{f})
\end{aligned}
$$

this implies that $\alpha_{X}$ is natural in $X$. Since $\alpha_{W}$ is injective, $\alpha_{W}(\zeta) \neq 0$. Let $\underline{g} \in \operatorname{rad}\left(\operatorname{End}_{\mathcal{C} / \mathcal{P}(\xi)}(W)\right)$. Then we have $g \in \operatorname{rad}\left(\operatorname{End}_{\mathcal{C}}(W)\right)$. Since $\zeta$ is an almost split sequence, we get that $\zeta g=0$. Consequently, $\alpha_{W}(\zeta)(g)={ }_{\varphi}\langle\zeta, g\rangle=$ $\varphi(\zeta g)=0$. This shows that $\alpha_{W}(\zeta)$ is almost vanishing on $\operatorname{End}_{\mathcal{C} / \mathcal{P}(\xi)}(W)$.

Conversely, let $\alpha: \xi \mathrm{xt}_{\mathcal{C}}^{1}(-, \tau W) \rightarrow D \operatorname{Hom}_{\mathcal{C} / \mathcal{P}(\xi)}(W,-)$ be a functorial monomorphism such that $\alpha_{W}(\zeta)$ is almost vanishing on $\operatorname{End}_{\mathcal{C} / \mathcal{P}(\xi)}(W)$. Then it is clear that $\zeta \neq 0$. Let $f: X \rightarrow W$ be a morphism in $\mathcal{C}^{b}(\bmod \Lambda)$ such that it is not a retraction. For any morphism $g: W \rightarrow L$, we have $f g \in \operatorname{rad}\left(\operatorname{End}_{\mathcal{C}}(W)\right)$, 
and hence, $f g=f \circ g \in \operatorname{rad}\left(\operatorname{End}_{\mathcal{C} / \mathcal{P}(\xi)}(W)\right)$. Thus $\alpha_{W}(\zeta)(f g)=0$, that is, $\left(D \operatorname{Hom}_{\mathcal{C} / \mathcal{P}(\xi)}(W, \underline{f}) \circ \alpha_{W}\right)(\zeta)=0$. By the following commutative diagram

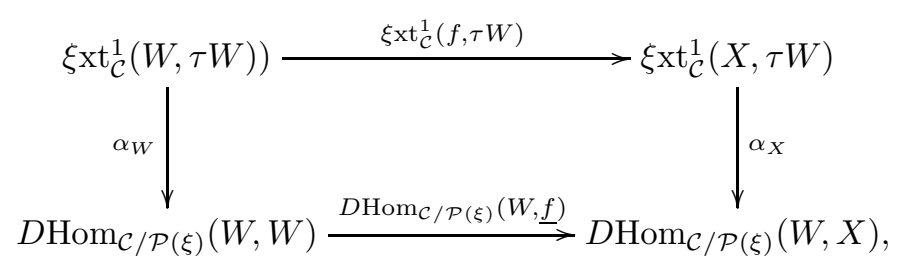

we get that $\left(\alpha_{X} \circ \xi \mathrm{xt}_{\mathcal{C}}^{1}(f, \tau W)\right)(\zeta)=0$. Since $\alpha_{X}$ is injective,

$$
\zeta f=\xi \operatorname{xt}_{\mathcal{C}}^{1}(f, \tau W)(\zeta)=0 .
$$

That is, $f$ factors through $V \rightarrow W$. On the other hand, we get by Lemma 3.8 that $\operatorname{End}_{\mathcal{C}}(\tau W)$ is local because $\operatorname{End}_{\mathcal{C}}(W)$ is local. Consequently, $\zeta$ is an almost split sequence. The proof of the theorem is completed.

Given two complexes $X$ and $Y$ in $\mathcal{C}^{b}(\bmod \Lambda)$. Then $D \operatorname{Hom}_{\mathcal{C} / \mathcal{P}(\xi)}(X, Y)$ is an $\operatorname{End}_{\mathcal{C}}(X)-\operatorname{End}_{\mathcal{C}}(Y)$-bimodule with multiplications defined, for $f \in \operatorname{End}_{\mathcal{C}}(X)$, $\theta \in D \operatorname{Hom}_{\mathcal{C} / \mathcal{P}(\xi)}(X, Y)$, and $g \in \operatorname{End}_{\mathcal{C}}(Y)$, by

$$
f \theta g: \operatorname{Hom}_{\mathcal{C} / \mathcal{P}(\xi)}(X, Y) \rightarrow E: \underline{h} \mapsto \theta(\underline{g h f}) .
$$

Similarly, $D \operatorname{Hom}_{\mathcal{C} / \mathcal{I}(\xi)}(X, Y)$ is an $\operatorname{End}_{\mathcal{C}}(X)-\operatorname{End}_{\mathcal{C}}(Y)$-bimodule.

Theorem 3.13. Let $W \in \mathcal{C}^{b}(\bmod \Lambda)$ be a complex with local endomorphism ring. Then the following assertions are equivalent.

(1) There exists an almost split sequence $\zeta: 0 \rightarrow \tau W \rightarrow V \rightarrow W \rightarrow 0$ in $\mathcal{C}^{b}(\bmod \Lambda)$ which is $C-E$ exact.

(2) The $\operatorname{End}_{\mathcal{C}}(W)$-socle of $\xi x t_{\mathcal{C}}^{1}(W, \tau W)$ is non-zero and there exists a functorial monomorphism $\alpha: \xi x t_{\mathcal{C}}^{1}(-, \tau W) \rightarrow D \operatorname{Hom}_{\mathcal{C} / \mathcal{P}(\xi)}(W,-)$.

(3) The $\operatorname{End}_{\mathcal{C}}(\tau W)$-socle of $\xi x t_{\mathcal{C}}^{1}(W, \tau W)$ is non-zero and there exists a functorial monomorphism $\beta: \xi x t_{\mathcal{C}}^{1}(W,-) \rightarrow D \operatorname{Hom}_{\mathcal{C} / \mathcal{I}(\xi)}(-, \tau W)$.

Where $\mathcal{C}$ denotes the bounded category $\mathcal{C}^{b}(\bmod \Lambda)$.

Proof. We will only prove the equivalence of assertions (1) and (2) by using an idea of the proof for [17, Th. 3.3].

Assume first that (1) holds. Then it follows from Proposition 3.2 that $W$ is not $\mathrm{C}-\mathrm{E}$ projective in $\mathcal{C}^{b}(\bmod \Lambda)$, and so by Theorem 3.12, there exists a functorial monomorphism $\alpha: \xi \operatorname{xt}_{\mathcal{C}}^{1}(-, \tau W) \rightarrow D \operatorname{Hom}_{\mathcal{C} / \mathcal{P}(\xi)}(W,-)$. Hence, $\zeta$ is a non-zero element in the $\operatorname{End}_{\mathcal{C}}(W)$-socle of $\xi \operatorname{xt}_{\mathcal{C}}^{1}(W, \tau W)$ since it is almost split.

Conversely, take a non-zero element $\zeta: 0 \rightarrow \tau W \rightarrow V \rightarrow W \rightarrow 0$ in the $\operatorname{End}_{\mathcal{C}}(W)$-socle of $\xi \operatorname{xt}_{\mathcal{C}}^{1}(W, \tau W)$. In particular, $\zeta$ is C-E exact and $W$ is not C-E projective. Let $\alpha: \xi \operatorname{xt}_{\mathcal{C}}^{1}(-, \tau W) \rightarrow D \operatorname{Hom}_{\mathcal{C} / \mathcal{P}(\xi)}(W,-)$ be a functorial monomorphism. Then $\alpha_{W}: \xi \operatorname{xt}_{\mathcal{C}}^{1}(W, \tau W) \rightarrow D \operatorname{End}_{\mathcal{C} / \mathcal{P}(\xi)}(W)$ is $\operatorname{End}_{\mathcal{C}}(W)$-linear since $\alpha$ is natural. Hence, $\theta=\alpha_{W}(\zeta)$ is a non-zero element 
of $D \operatorname{End}_{\mathcal{C} / \mathcal{P}(\xi)}(W)$, which is annihilated by $\operatorname{rad}\left(\operatorname{End}_{\mathcal{C}}(W)\right)$ because $\operatorname{End}_{\mathcal{C}}(W)$ is of finite $R$-length. If $\underline{f} \in \operatorname{rad}\left(\operatorname{End}_{\mathcal{C} / \mathcal{P}(\xi)}(W)\right)$, then $f \in \operatorname{rad}\left(\operatorname{End}_{\mathcal{C}}(W)\right)$ by Proposition 2.14, and so $\bar{\theta}(\underline{f})=(\theta f)\left(\underline{i d_{W}}\right)=0$. That is, $\theta=\alpha_{W}(\zeta)$ is almost vanishing on $\operatorname{End}_{\mathcal{C} / \mathcal{P}(\xi)}(W)$. By Theorem 3.12, $\zeta$ is an almost split sequence in $\mathcal{C}^{b}(\bmod \Lambda)$ which is $\mathrm{C}-\mathrm{E}$ exact. The proof of the theorem is completed.

Remark 3.14. It is clear that an almost split sequence $0 \rightarrow U \rightarrow V \rightarrow W \rightarrow 0$ in $\mathcal{C}^{b}(\bmod \Lambda)$ always is an almost split sequence in $\mathcal{C}(\bmod \Lambda)$.

Acknowledgements. This work is my postdoctoral research project at the University of Sherbrooke under the supervision by Professors Shiping Liu and David Smith. The author expresses his gratitude to the research group of algebra for the hospitality. The author is also supported partially by NSF of China (Nos. 11301240, 11201376, 11101197), and NSF of Gansu Province of China (No. 145RJZA079).

\section{References}

[1] I. Assem, D. Simson, and A. Skowronski, Elements of the Representation Theory of Associative Algebras Vol. I, London Mathematical Society Student Texts, 65. Cambridge University Press, Cambridge, 2006.

[2] M. Auslander, Comments on the functor Ext, Topology 8 (1969), 151-166.

[3] M. Auslander and I. Reiten, Representation theory of Artin algebras. III. Almost split sequences, Comm. Algebra 3 (1975), no. 3, 239-294.

[4] M. Auslander, I. Reiten, and S. Smalø, Representation Theory of Artin Algebras, Cambridge Univ. Press, Cambridge, 1995.

[5] M. Auslander and S. O. Smalø, Almost split sequences in subcategories, J. Algebra 69 (1981), no. 2, 426-454.

[6] M. Auslander and $\varnothing$. Solberg, Relative homology and representation theory I. Relative homology and homologically finite subcategories, Comm. Algebra 21 (1993), no. 9, 29953031.

[7] R. Bautista, M. J. Souto Salorio, and R. Zuazua, Almost split sequences of complexes of fixed size, J. Algebra, 287 (2005), no. 1, 140-168.

[8] P. Dräxler, I. Reiten, S. O. Smalø, and $\varnothing$. Solberg, Exact categories and vector space categories, Trans. Amer. Math. Soc. 351 (1999), no. 2, 647-682.

[9] E. E. Enochs, Cartan-Eilenberg complexes and resolutions, J. Algebra 342 (2011), 1639.

[10] P. Gabriel and A. V. Roiter, Representations of finite dimensional algebras, in: A. I. Kostrikin, I. V. Shafarevich (Eds.), Algebra VIII, in: Encyclopaedia Math. Sci., vol. 73, Springer, Berlin, 1992.

[11] D. Happel, On the derived category of a finite dimensional algebras, Comment. Math. Helv. 63 (1987), no. 3, 339-389.

[12] _ Triangulated categories in the representation theory of finite dimensional algebras, London Math. Soc. Lecture Note Series, vol. 119, Cambridge University Press, 1988.

[13] P. Jorgensen, Auslander-Reiten triangles in subcategories, J. K-Theory 3 (2009), no. 3, 583-601.

[14] M. Kleiner, Approximations and almost split sequences in homologically finite subcategories, J. Algebra 198 (1997), no. 1, 135-163.

[15] H. Krause and J. Le, The Auslander-Reiten formula for complexes of modules, Avd. Math. 207 (2006), no. 1, 133-148. 
[16] H. Lenzing and R. Zuazua, Auslander-Reiten duality for abelian categories, Bol. Soc. Mat. Mexicana 10 (2004), no. 2, 169-177.

[17] S. Liu, P. Ng, and C. Paquette, Almost split sequences and approximations, Algebr. Represent. Theory 16 (2013), no. 6, 1809-1827.

[18] P. Ng, Existence of Auslander-Reiten sequences in subcategories, J. Pure Appl. Algebra 215 (2011), no. 10, 2378-2384.

[19] S. Salarian and R. Vahed, Almost split sequences in the category of complexes of modules, J. Algebra Appl. 14 (2015), no. 3, 1550037, 15 pp.

[20] J.-L. Verdier, Des catégories dérivées des catégories abéliennes, Astérisque 239 (1996), $253 \mathrm{pp}$.

[21] P. Zhang, Monomorphism categories, cotilting theory, and Gorenstein-projective modules, J. Algebra 339 (2011), 181-202.

[22] X. Zhang and Z. Huang, On F-almost split sequences, Acta Math. Sin. (Engl. Ser.) 26 (2010), no. 6, 1149-1164.

Department of Mathematics

LANZHOU JiAOTONG UNIVERSITY

LANZHOU 730070, P. R. ChinA

E-mail address: yanggang@mail.lzjtu.cn 